\title{
Patterns of paediatric emergency admissions and predictors of prolonged hospital stay at the children emergency room, University of Calabar Teaching Hospital, Calabar, Nigeria
}

\author{
Callistus OA Enyuma ${ }^{1,2,3}$, Maxwell U Anah ${ }^{2,3}$, Amelia Pousson ${ }^{4}$, Olorunfemi G ${ }^{5}$, \\ Ibisomi L ${ }^{5}$, Abang B E ${ }^{3}$, Imoke $\mathrm{EJ}^{3}$
}

1. Division of Emergency Medicine, Faculty of Health Sciences, University of the Witwatersrand, Johannesburg, South Africa.

2. Department of Paediatrics, Faculty of Medicine, University of Calabar, Nigeria.

3. Department of Paediatrics, University of Calabar Teaching Hospital. Nigeria.

4. John Hopkins school of Medicine, Baltimore, Maryland, USA.

5. Division of Epidemiology and Biostatistics, School of Public health, University of the Witwatersrand,

Johannesburg, South Africa.

\section{Emails:}

Callistus OA Enyuma: drcarlenyuma@yahoo.com; Maxwell U Anah: maxejen@yahoo.com; Amelia Pousson: apousson@gmail.com; Olorunfemi G: drgbengafemi@yahoo.co.uk; Ibisomi, L: latifat.ibisomi@wits.ac.za; Abang B E: eccabrownr@gmail.com; Imoke EJ: imokejohn@yahoo.com

\begin{abstract}
Background: There is a high prevalence of paediatric emergency cases in less developed countries. However, prolonged hospital stay at emergency units may further overstretch the facilities.

Objective: To assess the patterns of presentations, services offered and predictors of a prolonged stay at the Children Emergency Room of a tertiary hospital in Southern Nigeria.

Methods: This prospective cross-sectional, study was conducted at the University of Calabar Teaching Hospital, Nigeria from $1^{\text {st }}$ January-31 ${ }^{\text {st }}$ December 2014. Socio-demographic and clinical characteristics of consecutively recruited children $(\mathrm{n}=633)$ were recorded in a proforma. Binary logistic regression was conducted to determine predictors of prolonged stay ( $>72$ hours)

Result: The median age of participants was $2(1-4.6)$ years. Three-fifths of children were admitted at off-hours and the commonest symptom was fever $(73.9 \%)$. About $16.4 \%(95 \% \mathrm{CI}: 13.6 \%-19.4 \%, \mathrm{n}=103 / 633)$ of the children had prolonged stay while those with sepsis had the longest mean stay (65.5 \pm 72.1 hours). Children admitted on account of Sickle cell disease (OR:11.2, 95\%CI:1.3-95.1, P-value $=0.03)$, Malaria (OR:10.7, 95\%CI:1.4-82.5, P-value $=0.02)$ or sepsis (OR:10.5, 95\%CI:1.3 - 82.7, P-value $=0.03)$ had higher odds of prolonged hospital stay. There was no significant difference in hospital stay among children admitted by the consultant as compared to other health personnel $(\mathrm{P}$-value $=0.08)$.

Conclusion: Prevention and proper management of Sickle cell disease and malaria reduces paediatric hospital stay in our environment. Paediatric emergency medicine should be re-organized to cater for high volume of off-hour admissions.

Keywords: Paediatric emergency, paediatric emergency admissions, predictors of prolonged stay, children emergency unit.

DOI: https://dx.doi.org/10.4314/ahs.v19i2.14

Cite as: Enyuma COA, Anah MU, Pousson A, Olorunfemi G, Ibisomi L, Abang BE, Imoke EJ. Patterns of paediatric emergency admissions and predictors of prolonged hospital stay at the Children Emergency Room, University of Calabar Teacbing Hospital, Calabar, Nigeria. Afri Health Sci.2019;19(2): 1910-1923.

https: / / dx.doi.org/10.4314/abs.v19i2.14

Corresponding author:

Callistus OA Enyuma,

Division of Emergency Medicine,

Charlotte Maxeke Johannesburg

Academic Hospital \& University

of the Witwatersrand,

Faculty of Health Sciences,

5 Jubilee Road, Parktown, Johannesburg,

2193, South Africa.

Cell: +27 635184154 and/or +234 8037026475 .

Email: drcarlenyuma@yahoo.com

\section{Introduction}

Emergency medicine is a dynamically complex and well-coordinated specialty. However, paediatric emergency sub-specialty is not yet well developed in most low and middle income countries (LMICs). In most LMICs, children emergency units (CHEU) are managed by generalist paediatricians with minimal expertise in paediatric emergency medicine. ${ }^{1-4}$ Thus, critical gaps in paediatric emergency care exist which may negatively impact outcomes. The situation is worse in Nigeria, where the ratio of paediatrician per population is low. ${ }^{2,5-7}$
\end{abstract}

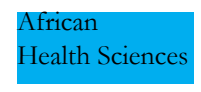

(C) 2019 Enyuma et al. Licensee African Health Sciences. This is an Open Access article distributed under the terms of the Creative commons Attribution License (https://creativecommons.org/licenses/BY/4.0), which permits unrestricted use, distribution, and reproduction in any medium, provided the original work is properly cited. 
The prevalence of preventable communicable diseases (such as malaria, pneumonia and diarrhoeal diseases) is high in sub-Saharan African Countries. ${ }^{8-12}$ The aforementioned diseases are commoner among the under-fives, who account for over $70 \%$ of paediatric emergency admissions. ${ }^{11,13-15}$ In addition to the high burden of paediatric infectious disease, there is at present an increasing incidence of non-communicable and hereditary diseases and their complications. This includes sickle cell disease, diabetes mellitus and hypertension. ${ }^{2,16-20}$

Mortality rates in the Children Emergency Room (CHER) in Nigeria ranged from 2-17.5\%, with about $40 \%$ of deaths occurring in the first 24 hours of admission., ${ }^{211,12,15,18,21-25}$ Late presentation, delayed interventions, financial constraints, unavailability of life saving equipment and inadequate support services may play a role in this observed mortality pattern. . $^{3,12,18,23,26}$

At CHER, children are typically triaged, resuscitated and then transferred to either paediatric lying-in ward or discharged to be followed up at children out-patient's clinic(CHOP). ${ }^{24}$ To prevent overcrowding in CHER, target length of stay (LOS) for patient disposition is set at 48-72 hours in Nigeria and 4-8 hours in most developed countries. ${ }^{21,27,28}$ However, factors such as patient inflow, CHER admission and discharge processes, as well as hospital bottlenecks and level of facilities may contribute to prolonged hospital stay. Prolonged hospital stay may cause: socio-cognitive problems to the child; socio-economic burdens to the parents and increased constraints on the health facility resources. ${ }^{27-29}$

Although pattern of presentations and predictors of paediatric hospital stay has been reported in the high income countries $^{30-33}$, little is known currently in our environment.

We therefore, conducted this study to assess the current pattern of presentations and outcomes, including predictors of prolonged stay at CHER of the University of Calabar Teaching Hospital (UCTH), Southern Nigeria to inform policy and improve paediatric emergency practice in our environment.

\section{Materials and methods}

This was a prospective cross-sectional study of consenting consecutive children admitted to the CHER of UCTH, Calabar, Southern Nigeria from 1st January to $31^{\text {st }}$ December 2014.
The children emergency unit has continuously been reorganized for improved performance. It has a triage area, two consulting areas, a resuscitation area, a procedure area, a short stay ward of 35 beds and in addition a diarrhoea treatment and training unit (DTTU) of 6 beds with carefully laid out patients flow plan.

Since 2013, a resident doctor triages all newly presented children using the South African Triage Score. ${ }^{34}$ A consultant paediatrician is permanently posted to the unit, and also reviews all patients within 2 hours of admission during week days. All paediatric subspecialty consultants conduct ward rounds and take calls in CHER. Hand-over rounds are conducted at 8:00 am and $4 \mathrm{pm}$ daily and mortalities are reviewed weekly. The unit policy is to see all patients within 15 minutes of arrival and dispose within 72 hours except the unstable patients that need continued care.

After initial stabilization, the parent or guardian of consecutive patients was approached for recruitment by the research team. , Informed consent was obtained from consecutively recruited participants. Those that declined consent were excluded from the study. Information was continually retrieved from patients' file and entered into the research proforma by the paediatric residents, who were trained about data extraction and blinded to the study objectives. The principal investigator randomly cross checked the data entry with the records in the actual file. Missing data was cross-checked from the original files.

The information extracted included; time of arrival and time of review by medical personnel, cadre of doctor that first saw the patient, immunization status, presenting complaint, treatment instituted at home, signs elicited, working diagnosis, investigations requested, treatment received, duration of stay (calculated from time of admission and time of discharge in hours) and discharge diagnosis. Diagnosis was based on International classification of Diseases (Tenth revision) (ICD-10). The outcome measured were discharges, duration of stay, transferred out, Discharged against medical advice (DAMA), absconded and death. Daily updates of the records of each patient were done until the patient was discharged from CHER. Ethical approval was obtained from the hospital health and research ethics committee (HREC). There was no intervention and the information recorded in the proforma was anonymized. 


\section{Data analysis}

Data was entered into excel spread sheet (microsoft $\AA$ Excel ${ }^{\circledR} 2010$ ) from the proforma and exported to Stata version 14 (Stata corp ${ }^{\circledR} 2015$ College station TX) Statistical software. Descriptive statistics was conducted and categorical variables were expressed as frequencies, percentages and charts. Normally distributed continuous variables were presented as means and standard deviations (SD) while non-normally distributed continuous variables were presented as median and interquartile range (IQR). Length of hospital stay was categorized into prolonged ( $\geq 72$ hours) and non-prolonged stay ( $<72$ hours). The categorical clinical and socio-demographic characteristics were compared based on hospital stay classifications using a Pearson's Chi-square (Fishers exact test was utilized if the expected cell was less than 5). Student's t-test and Mann Whitney U was respectively used to determine an association between normally and non-normally distributed continuous variables and the duration of stay (prolonged/non-prolonged). Analysis of variance was used to compare the mean length of stay among groups. Post hoc Bonferroni test was conducted for the statistically significant ANOVA values. Univariable binary logistic regression was conducted between explanatory variables and the outcome (prolonged or non-prolonged hospital stay). Multivariable binary logistic regression was then conducted with explanatory variables that had P-value $<0.2$ from univariable analysis. Age and cadre of admitting staff were however pre-determined a priori based on literature and the experience of the researchers. Adjusted odds ratio with $95 \%$ confidence intervals was reported for predictors of prolonged hospital stay. Two-tailed test of hypothesis was assumed. Statistically significant level was set at $\mathrm{P}$-value $<0.05$.

\section{Results}

\section{Study population and socio-demographic character-} istics:

Of the 2,023 children that presented to CHEU during the study period, $633(76.4 \%)$ were admitted into CHER and recruited into the study while $195(23.6 \%)$ were admitted into DTTU and 1,195 (59.1\%) went home (See Figure 1) Over two-thirds $(410 / 633 ; 77.5 \%)$ of the patients were five years and below, and the median age (interquartile range) was $2(1-4.6)$ years. The male to female ratio was 1.3:1. (Table 1)

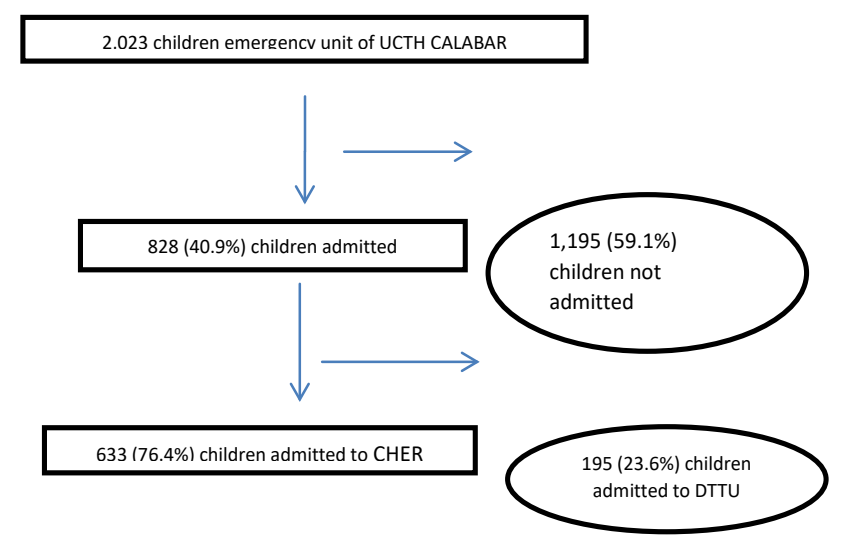

Figure 1: Flow chart of the study participants. 
Table 1: Comparison of the socio-demographic and clinical characteristics of children by duration of stay in the hospital

\begin{tabular}{|c|c|c|c|c|c|c|}
\hline Characteristics & Less 72 hours & $\begin{array}{l}\text { Prolonged stay } \\
\text { ( }>72 \text { hours) }\end{array}$ & Total & $95 \%$ CI & $\begin{array}{l}\text { Test- } \\
\text { statistics }\end{array}$ & P-values \\
\hline $\begin{array}{l}\text { Age (years) } \\
\text { (median (IQR)) }\end{array}$ & $2(1.0-4.6)$ & $(1.0-4.7)$ & $2(1-4.6)$ & & $0.22^{\wedge}$ & 0.8224 \\
\hline$\leq 1$ & $119(22.5)$ & $25(24.0)$ & $144(22.8)$ & $19.6-26.2$ & $0.67^{\#}$ & 0.881 \\
\hline $1-5$ & $291(55.0)$ & $59(56.7)$ & $350(55.3)$ & $51.4-59.1$ & & \\
\hline $6-12$ & $91(17.2)$ & $16(15.4)$ & $107(16.9)$ & $14.2-20.0$ & & \\
\hline$>12$ & $28(5.3)$ & $4(3.9)$ & $32(5.1)$ & $3.6-7.1$ & & \\
\hline \multicolumn{7}{|l|}{ Gender } \\
\hline Female & $237(44.9)$ & $37(35.6)$ & $274(43.4)$ & $39.5-47.3$ & $3.07^{\#}$ & 0.080 \\
\hline \multirow{2}{*}{\multicolumn{7}{|c|}{$\begin{array}{l}\text { Male } \\
{ }^{\ddagger} \text { Tribe/Ethnicity }\end{array}$}} \\
\hline & & & & & & \\
\hline South & $117(22.1)$ & $21(20.2)$ & $138(21.8)$ & $18.7-25.2$ & $5.55^{\#}$ & 0.235 \\
\hline Central & $80(15.1)$ & $21(20.2)$ & $101(16.0)$ & $13.3-19.0$ & & \\
\hline North & $59(11.2)$ & $14(13.5)$ & $73(11.5)$ & $9.3-14.3$ & & \\
\hline Akwa Ibom & $161(30.4)$ & $35(33.7)$ & $196(31.0)$ & $27.5-34.7$ & & \\
\hline Others & $112(21.2)$ & $13(12.5)$ & $125(19.8)$ & $16.8-23.0$ & & \\
\hline \multicolumn{7}{|l|}{ Period of presentation } \\
\hline Morning & $238(45.0)$ & $39(37.5)$ & $277(43.8)$ & $39.9-47.7$ & $3.08^{\#}$ & 0.214 \\
\hline Afternoon & $201(38.0)$ & $49(47.1)$ & $250(39.5)$ & $35.7-43.4$ & & \\
\hline Night & $90(17.0)$ & $16(15.4)$ & $106(16.8)$ & $14.0-19.9$ & & \\
\hline \multicolumn{7}{|l|}{${ }^{\Omega}$ Mode of referral } \\
\hline Self & $420(79.4)$ & $81(77.9)$ & $501(79.2)$ & $75.8-82.1$ & & $0.891^{*}$ \\
\hline PHC & $3(0.57)$ & $0(0.0)$ & $3(0.5)$ & $0.2-1.5$ & & \\
\hline SHC & $20(3.8)$ & $5(4.8)$ & $25(4.0)$ & $2.7-5.8$ & & \\
\hline CHOP & $85(16.1)$ & $18(17.3)$ & $103(16.3)$ & $13.6-19.4$ & & \\
\hline Others & $1(0.2)$ & $0(0.00)$ & $1(0.16)$ & $0.0-1.1$ & & \\
\hline \multicolumn{7}{|l|}{ Prior treatment } \\
\hline Home remedy & $266(50.3)$ & $55(52.9)$ & $321(50.7)$ & $46.8-54.6$ & & $0.022 *$ \\
\hline Health facility & $78(14.7)$ & $16(15.4)$ & $94(14.9)$ & $12.3-17.9$ & & \\
\hline Alternative care facility & $0(0.0)$ & $1(0.96)$ & $1(0.2)$ & $0.0-1.1$ & & \\
\hline Multiple & $162(30.6)$ & $22(21.2)$ & $184(29.1)$ & $3.7-7.3$ & & \\
\hline None & $23(4.4)$ & $10(9.6)$ & $33(5.2)$ & $25.7-32.7$ & & \\
\hline \multicolumn{7}{|l|}{ Admitting Doctor } \\
\hline House officer & $106(20.0)$ & $18(17.3)$ & $124(19.6)$ & $16.7-22.9$ & $2.80^{\#}$ & 0.424 \\
\hline Junior resident & $260(49.2)$ & $50(48.1)$ & $310(49.0)$ & $45.1-52.9$ & & \\
\hline Senior resident & $145(27.4)$ & $29(27.9)$ & $174(27.5)$ & $24.1-31.1$ & & \\
\hline Consultant & $18(3.4)$ & $7(6.7)$ & $25(4.0)$ & $2.7-5.8$ & & \\
\hline $\begin{array}{l}\text { Duration of illness (Hours) } \\
\text { (Median (IQR)) }\end{array}$ & $4(2-5)$ & $4.5(3-5)$ & $4(2-5)$ & & $0.06^{\wedge}$ & 1.891 \\
\hline$\leq 12$ & $67(12.7)$ & $10(9.6)$ & $77(12.2)$ & $9.8-15.0$ & $4.06^{\#}$ & 0.397 \\
\hline $13-24$ & $95(18.0)$ & $15(14.4)$ & $110(17.4)$ & $14.6-20.5$ & & \\
\hline $25-48$ & $76(14.4)$ & $11(10.6)$ & $87(13.7)$ & $11.3-16.7$ & & \\
\hline $49-72$ & $76(14.4)$ & $16(15.4)$ & $92(14.5)$ & $12.0-17.5$ & & \\
\hline$>72$ & $215(40.6)$ & $52(50.0)$ & $267(42.2)$ & $38.4-46.1$ & & \\
\hline \multicolumn{7}{|l|}{ Diagnosis at discharge } \\
\hline Malaria & $128(24.2)$ & $33(31.7)$ & $161(25.4)$ & $22.2-29.0$ & 9.46 & 0.149 \\
\hline Sepsis/Meningitis & $51(9.6)$ & $13(12.5)$ & $64(10.1)$ & $8.0-12.7$ & & \\
\hline Respiratory pathology & $220(41.6)$ & $40(38.5)$ & $260(41.1)$ & $37.3-45.0$ & & \\
\hline Sickle Cell Anaemia & $44(8.3)$ & $10(9.6)$ & $54(8.5)$ & $6.6-11.0$ & & \\
\hline${ }^{\alpha}$ GIT pathology & $45(8.5)$ & $7(6.7)$ & $52(8.2)$ & $6.3-10.6$ & & \\
\hline Surgical/ Burns & $29(5.5)$ & $1(1.0)$ & $30(4.7)$ & $3.3-6.7$ & & \\
\hline \multirow{2}{*}{\multicolumn{7}{|c|}{ Outcomes }} \\
\hline & & & & & & \\
\hline Discharge & $417(78.9)$ & $1(0.96)$ & $418(66.0)$ & $62.2-69.6$ & & $<0.001 *$ \\
\hline Transferred & $85(16.1)$ & $0(0.0)$ & $85(13.4)$ & $11.0-16.3$ & & \\
\hline DAMA & $21(4.0)$ & $0(0.0)$ & $21(3.3)$ & $2.2-5.0$ & & \\
\hline Absconded & $3(0.6)$ & $0(0.0)$ & $3(0.5)$ & $0.2-1.5$ & & \\
\hline Died & $3(0.6)$ & $0(0.0)$ & $3(0.5)$ & $0.2-1.5$ & & \\
\hline Prolong stay & $0(0.0)$ & $103(99.0)$ & $103(16.3)$ & $13.6-19.4$ & & \\
\hline
\end{tabular}




\section{Clinical characteristics and presentations:}

About one-third ( $n=187 / 633 ; 29.5 \%)$ of participants presented within 24 hours of illness and almost half $(\mathrm{n}=277 / 633 ; 43.8 \%)$ presented during the morning shift. Majority $(501 / 633 ; 79.2 \%)$ were not formally referred. About half ( $\mathrm{n}=321 / 633 ; 50.7 \%$ ) had received home remedy and only about 15\% (94/633) previously accessed peripheral health care facilities in current illness. (Table 1) Almost all participants $(n=627,99.1 \%)$ had completed routine immunizations for age and about half (49.0\%, 310/633) were first seen by Junior Resident doctors. (Table 1)

The common presenting symptoms were: history of fe$\operatorname{ver}(\mathrm{n}=468 / 633 ; 73.9 \%)$, and cough $(\mathrm{n}=239 / 633 ; 37.7 \%)$ though, some presented with multiple symptoms. (Figure 1)

The common diagnoses at admission were: respiratory tract infections $(n=341,53.7 \%)$, and malaria $(n=332$, $52.4 \%$ ). (Figure 2) Their final diagnoses did not differ significantly. (Table 1)

\section{Hospital interventions and outcomes:}

Majority ( $\mathrm{n}=430 / 633 ; 67.9 \%$ ) of the patients received intervention within 30 minutes of arrival. At discharge, four-fifth $(82.3 \% ; n=521 / 633)$ of patients received antibiotics though only $22 \%(n=182 / 633)$ had a blood culture result. Forty $(6.3 \% ; n=40 / 633)$ children received blood transfusion, the majority $(31 / 40 ; 77.5 \%)$ were due to anaemia from severe malaria. (Table 2 )

Table 2: Distribution of the various interventions received by the children at the Children emergency Room of UCTH, Calabar, Nigeria

\begin{tabular}{|c|c|}
\hline Interventions , $\mathrm{N}=633$ & Frequency (\%) \\
\hline \multicolumn{2}{|l|}{ Antibiotics } \\
\hline Parenteral & $480(75.8)$ \\
\hline Oral & $41(6.5)$ \\
\hline \multicolumn{2}{|l|}{ Fluids } \\
\hline IVF & $211(33.3)$ \\
\hline ORT & $43(6.8)$ \\
\hline ORT/IVF & $35 \quad(5.5)$ \\
\hline Resomal & $1(0.2)$ \\
\hline $10 \%$ dextrose bolus & $4 \quad(0.6)$ \\
\hline \multicolumn{2}{|l|}{ Asthmatic treatment } \\
\hline Bronchodilator/steroid & $92(14.5)$ \\
\hline Hydrocortisone./salbutamol nebules & $64(69.6)$ \\
\hline Prednisolone/salbutamol nebules & $4(4.4)$ \\
\hline Hydrocortisone/Aminophylline & $2(2.2)$ \\
\hline Steam inhalation & $22(23.9)$ \\
\hline Oxygen & $56(8.8)$ \\
\hline $\mathrm{NaHco} 3$ & $8(1.3)$ \\
\hline Suction & $6(0.9)$ \\
\hline Transfusion & $40(6.3)$ \\
\hline Diazepam & $43(6.8)$ \\
\hline
\end{tabular}

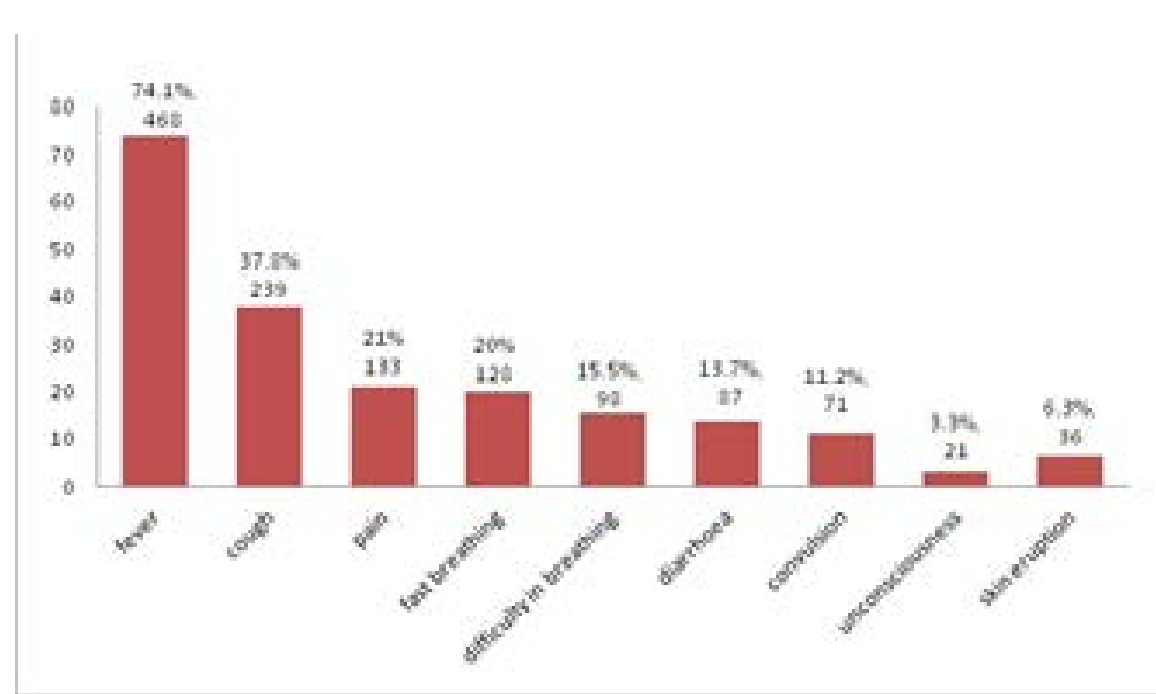

Figure 2: Distribution of presenting symptoms of children admitted to the children emergency Room of UCTH, Calabar, Nigeria 
The mortality rate in this study was low $(n=3 / 633 ; 0.5 \%)$. (Table 1)

\section{Length of stay:}

The minimum and maximum length of stay was 0.5 hours and 456 hours respectively while the median length of stay (interquartile range) was 48 (24-72) hours. About 16.4\% (95\%CI:13.6\% - 19.4\%, $n=103 / 633)$ of children had prolonged hospital stay and children with sepsis/ meningitis had the longest mean hospital stay (65.5 \pm 72.1 hours) followed by malaria (61.5 \pm 36.9 hours) and sickle cell anaemia (59.0 \pm 35.9 hours). While surgical cases (30.9 \pm 26.4 hours) had the least hospital stay. Children that had prior treatment at non-orthodox facilities had highest mean hospital stay (216.0 \pm 0.0 hours). (Table $3 \mathrm{~A}$ ) Post hoc Bonferroni test of pair-wise difference in mean length of stay is as shown in Table 3B below

Table 3A. The mean length of stay of children admitted to CHER

\begin{tabular}{|c|c|c|}
\hline Characteristics & (Mean \pm SD) hours & P-value \\
\hline \multicolumn{3}{|l|}{ Age (years) } \\
\hline$\leq 1$ & $60.3 \pm 37.1$ & $0.3862^{\wedge}$ \\
\hline $1-5$ & $54.5 \pm 43.5$ & \\
\hline $6-12$ & $52.6 \pm 35.1$ & \\
\hline$>12$ & $51.9 \pm 39.9$ & \\
\hline \multicolumn{3}{|l|}{ Gender } \\
\hline male & $57.2 \pm 43.9$ & $0.2117^{\#}$ \\
\hline Female & $53.1 \pm 35.7$ & \\
\hline \multicolumn{3}{|l|}{${ }^{*}$ Tribe/Ethnicity } \\
\hline South & $51.1 \pm 37.7$ & $0.2760^{\wedge}$ \\
\hline Central & $62.1 \pm 38.0$ & \\
\hline North & $53.1 \pm 39.6$ & \\
\hline Akwa Ibom & $57.0 \pm 48.4$ & \\
\hline \multirow{2}{*}{\multicolumn{3}{|c|}{ Period of presentation }} \\
\hline & & \\
\hline Morning & $53.8 \pm 42.1$ & $0.4949^{\wedge}$ \\
\hline Afternoon & $57.7 \pm 40.3$ & \\
\hline Night & $53.9 \pm 37.0$ & \\
\hline \multicolumn{3}{|l|}{${ }^{{ }^{S 2}}$ Mode of referral } \\
\hline Self & $55.8 \pm 41.4$ & $0.9897^{\wedge}$ \\
\hline PHC & $48.0 \pm 0.0$ & \\
\hline SHC & $54.1 \pm 46.5$ & \\
\hline CHOP & $54.1 \pm 35.6$ & \\
\hline Others & $48.00 \pm 0.0$ & \\
\hline \multicolumn{3}{|l|}{ Prior treatment } \\
\hline Home remedy & $55.0 \pm 32.3$ & $<0.0001^{\wedge}$ \\
\hline Health facility & $60.1 \pm 42.9$ & \\
\hline Alternative carefacility & $216.0 \pm 0.0$ & \\
\hline Multiple & $48.6 \pm 38.4$ & \\
\hline None & $78.5 \pm 81.5$ & \\
\hline \multicolumn{3}{|l|}{ Admitting Doctor } \\
\hline House officer & $53.0 \pm 52.3$ & $0.8334^{\wedge}$ \\
\hline Junior resident & $55.1 \pm 38.3$ & \\
\hline Senior resident & $57.2 \pm 33.0$ & \\
\hline Consultant & $57.9 \pm 50.8$ & \\
\hline \multicolumn{3}{|c|}{ Duration of illness (Hours) } \\
\hline$\leq 12$ & $47.2 \pm 37.9$ & $0.1962^{\wedge}$ \\
\hline $13-24$ & $55.4 \pm 35.1$ & \\
\hline $25-48$ & $52.1 \pm 37.8$ & \\
\hline $49-72$ & $54.4 \pm 34.0$ & \\
\hline \multirow{2}{*}{\multicolumn{3}{|c|}{ Diagnosis at discharge }} \\
\hline & & \\
\hline Malaria & $61.5 \pm 36.9$ & $0.0001^{\wedge}$ \\
\hline Sepsis/Meningitis & $65.5 \pm 72.1$ & \\
\hline Respiratory pathology & $52.8 \pm 33.3$ & \\
\hline Sickle Cell Anaemia & $59.0 \pm 35.9$ & \\
\hline${ }^{\alpha}$ GIT pathology & $53.0 . \pm 37.3$ & \\
\hline \multirow{2}{*}{\multicolumn{3}{|c|}{ Outcomes }} \\
\hline & & \\
\hline Early discharge & $45.0 \pm 22.0$ & $<0.0001^{\wedge}$ \\
\hline Transferred & $39.3 \pm 24.5$ & \\
\hline DAMA & $17.9 \pm 14.4$ & \\
\hline Absconded & $17.0 \pm 6.0$ & \\
\hline Died & $18.7 \pm 25.6$ & \\
\hline Prolong stay & $120.5 \pm 48.6$ & \\
\hline
\end{tabular}


Table 3B. Pairwise Post Hoc Bonferroni test of mean stay among categories of variables

\begin{tabular}{|c|c|c|c|c|c|c|}
\hline \multicolumn{7}{|c|}{ Prior treatment } \\
\hline & $\begin{array}{l}\text { Home } \\
\text { remedy/ } \\
\text { Alternative } \\
\text { care } \\
\text { facility }\end{array}$ & Health facility & $\begin{array}{l}\text { Multiple } \\
\text { Treatment }\end{array}$ & None & & \\
\hline $\begin{array}{l}\text { Home remedy/ } \\
\text { Alternative care } \\
\text { facility }\end{array}$ & 1.00 & & & & & \\
\hline Health facility & 1.000 & 1.00 & & & & \\
\hline $\begin{array}{l}\text { Multiple } \\
\text { Treatment }\end{array}$ & 0.383 & 0.146 & 1.00 & & & \\
\hline None & $0.011^{*}$ & 0.144 & $0.001 *$ & 1.00 & & \\
\hline \multicolumn{7}{|c|}{ Diagnosis at discharge } \\
\hline & Malaria & Sepsis/Meningitis & $\begin{array}{l}\text { Respiratory } \\
\text { pathology }\end{array}$ & $\begin{array}{l}\text { Sickle cell } \\
\text { Anaemia }\end{array}$ & $\begin{array}{l}\text { GIT } \\
\text { Pathology }\end{array}$ & $\begin{array}{l}\text { Surgical } \\
\text { /Burns/others }\end{array}$ \\
\hline Malaria & 1.00 & & & & & \\
\hline Sepsis/Meningitis & 1.000 & 1.00 & & & & \\
\hline $\begin{array}{l}\text { Respiratory } \\
\text { pathology }\end{array}$ & 0.439 & 0.335 & 1.00 & & & \\
\hline $\begin{array}{ll}\text { Sickle } & \text { Cell } \\
\text { Anaemia } & \end{array}$ & 1.000 & 1.000 & 1.000 & 1.00 & & \\
\hline${ }^{\alpha}$ GIT pathology & 1.000 & 1.000 & 1.000 & 1.000 & 1.00 & \\
\hline $\begin{array}{l}\text { Surgical/ Burns/ } \\
\text { Others }\end{array}$ & $0.001 *$ & $0.001 *$ & $0.016^{*}$ & $0.010^{*}$ & 0.121 & 1.00 \\
\hline \multicolumn{7}{|c|}{ Outcomes } \\
\hline & $\begin{array}{l}\text { Early } \\
\text { Discharge }\end{array}$ & Transferred & DAMA & Absconded & Died & Prolong Stay \\
\hline Early Discharge & 1.00 & & & & & \\
\hline Transferred & 1.000 & 1.00 & & & & \\
\hline DAMA & $0.001^{*}$ & $0.029^{*}$ & 1.00 & & & \\
\hline Absconded & 1.000 & 1.000 & 1.000 & 1.00 & & \\
\hline Died & 1.000 & 1.000 & 1.000 & 1.000 & 1.00 & \\
\hline Prolong stay & $<0.001^{*}$ & $0.001 *$ & $0.001 *$ & $0.001^{*}$ & $0.001^{*}$ & 1.00 \\
\hline
\end{tabular}

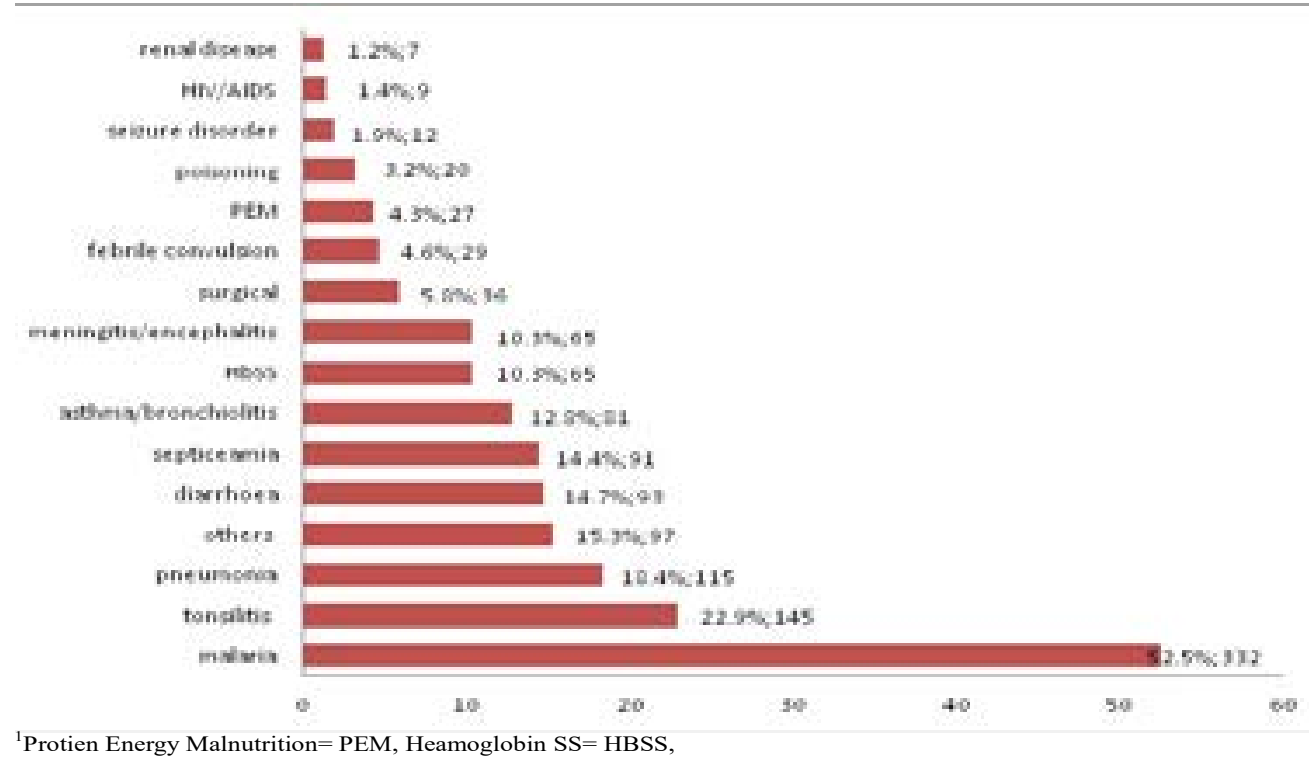

Figure 3: Distribution of diagnosis of children admitted to the children emergency Room of UCTH, Calabar, Nigeria 


\section{Predictors of prolonged hospital stay:}

At univariable analysis, treatments before presentation and diagnosis at discharge were associated with prolonged stay. (Table 4)

From multivariable analysis, children that had prior multiple treatments, home or herbal remedy or that visited another health facility before presentation had 59\%, 74\% and $61 \%$ lesser chance of staying beyond 72 hours as compared to children that did not. Furthermore, children whose final diagnosis were SCA, malaria, sepsis or respiratory illness had 11.2-,10.7-,10.2- , and 7.6-fold increased odds of having prolonged stay as compared to children with surgical conditions(For sickle cell disease: OR:11.2, 95\%CI:1.3-95.1, P-value $=0.03$; Malaria OR:10.7, 95\%CI:1.4-82.5, P-value $=0.02$; sepsis:OR:10.5,
95\%CI:1.3 - 82.7, P-value $=0.03)$. Although, participants first seen by consultant had about 3-fold increased odds of prolonged stay as compared to children first seen by house officers, this relationship did not reach statistical significance at 95\% confidence interval (Adj OR: 2.57; 95\%CI: $0.90-7.31 ;$ P-value $=0.08)$. Table 3 also showed that children admitted by Consultants had the highest mean length of stay (57.9 \pm 50.8 hours) but the difference was not statistically significant $(\mathrm{P}$-value $=0.8334)$. Also, admissions during the afternoon shift were about 1.6 times more likely to have prolonged stay as compared to morning shift admissions. This relationship was not statistically significant $(\mathrm{P}$-value $=0.06)$. There was no statistically significant difference in the odds of having prolonged hospital stay and the age of the children. (Table 4)

Table 4: Univariable and multivariable logistic regression analysis of predictors of prolonged hospital stay in the Children Emergency Room, UCTH, Calabar.

\begin{tabular}{|c|c|c|c|c|c|c|}
\hline \multirow{2}{*}{$\begin{array}{l}\text { Univariable analysis } \\
\text { Factor }\end{array}$} & \multirow[b]{2}{*}{ COR } & \multirow[b]{2}{*}{$95 \%$ CI } & \multirow[b]{2}{*}{ P-value } & \multicolumn{2}{|c|}{ Multivariable analysis } & \multirow[b]{2}{*}{ P-value } \\
\hline & & & & Adj OR & $95 \%$ CI & \\
\hline \multicolumn{7}{|l|}{ Age (years) } \\
\hline Above 12 & 1.00 & Ref & Ref & 1.00 & Ref & Ref \\
\hline$<1$ & 1.47 & $0.47-4.57$ & 0.51 & 1.40 & $0.42-4.70$ & 0.59 \\
\hline $1-5$ & 1.42 & $0.48-4.20$ & 0.53 & 1.28 & $0.40-4.08$ & 0.68 \\
\hline $6-12$ & 1.23 & $0.38-3.98$ & 0.73 & 1.03 & $0.30-3.47$ & 0.96 \\
\hline \multicolumn{7}{|l|}{ Shift of admission (hours) } \\
\hline Morning (8.00- 14.00$)$ & 1.00 & Ref & Ref & 1.00 & Ref & Ref \\
\hline Afternoon (14.00-22.00) & 1.49 & $0.94-2.36$ & 0.09 & 1.60 & $0.99-2.60$ & $0.06 *$ \\
\hline Night $(22.00-8.00)$ & 1.08 & $0.58-2.04$ & 0.80 & 1.28 & $0.65-2.50$ & 0.47 \\
\hline \multicolumn{7}{|l|}{ Cadre of admitting doctor } \\
\hline House officer/interns & 1.00 & Ref & Ref & 1.00 & Ref & Ref \\
\hline Junior Resident & 1.13 & $0.63-2.03$ & 0.68 & 1.11 & $0.61-2.02$ & 0.74 \\
\hline Senior Resident & 1.18 & $0.62-2.23$ & 0.62 & 1.21 & $0.62-2.36$ & 0.57 \\
\hline Consultant & 2.29 & $0.84-6.26$ & 0.11 & 2.57 & $0.90-7.31$ & $0.08 *$ \\
\hline \multicolumn{7}{|c|}{ Previous treatment before presentation } \\
\hline None & 1.00 & Ref & Ref & 1.00 & Ref & Ref \\
\hline Home or herbal remedy/alt & 0.48 & $0.22-1.07$ & $0.07 *$ & 0.41 & $0.18-0.95$ & $0.04 *$ \\
\hline Health facility & 0.47 & $0.19-1.18$ & 0.11 & 0.39 & $0.15-1.02$ & $0.05^{*}$ \\
\hline Multiple treatment & 0.31 & $0.13-0.74$ & $0.008^{*}$ & 0.26 & $0.11-0.65$ & $0.004 *$ \\
\hline \multicolumn{7}{|l|}{ Discharge diagnosis } \\
\hline Surgical/Others & 1.00 & Ref & Ref & 1.00 & Ref & Ref \\
\hline Malaria & 10.57 & $1.40-79.71$ & $0.02 *$ & 10.67 & $1.38-82.49$ & $0.02 *$ \\
\hline Sepsis/Meningitis & 10.45 & $1.31-83.24$ & $0.03 *$ & 10.16 & $1.25-82.71$ & $0.03 *$ \\
\hline Respiratory pathology & 7.45 & $0.996-55.75$ & 0.05 & 7.58 & $0.99-58.22$ & 0.05 \\
\hline Sickle cell anaemia & 9.32 & $1.14-76.03$ & $0.04 *$ & 11.23 & $1.32-95.14$ & $0.03 *$ \\
\hline Gastrointestinalpathology & 6.38 & $0.75-54.08$ & 0.09 & 6.29 & $0.72-54.72$ & 0.10 \\
\hline \multicolumn{7}{|l|}{ Gender } \\
\hline Male & 1.00 & Ref & Ref & & & \\
\hline Female & 0.68 & $0.44-1.05$ & 0.08 & & & \\
\hline \multicolumn{7}{|c|}{ Indigenous Districts in Cross River state and others } \\
\hline South district & 1.00 & Ref & Ref & & & \\
\hline Central district & 1.46 & $0.75-2.85$ & 0.27 & & & \\
\hline North district & 1.32 & $0.63-2.79$ & 0.46 & & & \\
\hline Akwa Ibom State & 1.21 & $0.67-2.19$ & 0.53 & & & \\
\hline Others & 0.65 & $0.31-1.35$ & 0.25 & & & \\
\hline \multicolumn{7}{|c|}{ Duration of illness before presentation } \\
\hline$<12$ & 1.00 & Ref & Ref & & & \\
\hline $13-24$ & 1.06 & $0.45-2.50$ & 0.90 & & & \\
\hline $25-48$ & 0.97 & $0.39-2.43$ & 0.95 & & & \\
\hline $49-72$ & 1.41 & $0.60-3.32$ & 0.43 & & & \\
\hline$>72$ & 1.62 & $0.78-3.36$ & 0.20 & & & \\
\hline
\end{tabular}




\section{Discussion}

This study sought to assess the patterns of presentations, morbidity and mortality, and the predictors of prolonged stay at CHER of the University of Calabar Teaching Hospital, Calabar Southern Nigeria from 1st January to 31st December, 2014.

\section{Admission pattern and presentations}

We found that about two-thirds of admitted children were under-fives and more males than females were admitted This admission pattern is typical of age-related morbidity pattern in LMICs. ${ }^{211-13,15,18,21-23,35}$ In comparison to older children, under-fives are more vulnerable to infectious diseases, the predominant cause of emergency admissions in Nigeria. ${ }^{11,13,14}$

Although early presentation can improve prognosis, only one-third of the admitted children presented early (within 24 hours of illness). From previous studies, the reasons for late presentations at paediatric emergency units includes poor identification of early sign of disease severity by care givers; exorbitant cost of hospital treatment as patient pay out of pocket, poor health seeking behaviour and beliefs in remedies with unproven efficacy. ${ }^{36,37}$ Thus, it is not surprising that prior to their presentation, about half of the study participants had received home remedy.

Furthermore, majority $(79.2 \%)$ of admitted patients came to the hospital without formal referral while a few $(16.3 \%)$ were referred from CHOP of our hospital. Our finding is comparable to the study by Linden et al who reported a self-referral of $61 \%{ }^{38}$. Only one-third of the self-referrals presented during morning shift in our study. This is similar to findings by Opunabo et al in Port Harcourt, Nigeria. ${ }^{29}$ The high prevalence of self-referrals at our centre may be because acutely-ill children present a challenge and dilemma to the parents, who reflexly or in desperation, sought for immediate medical assistance at CHER, even at odd hours. However, studies have shown that majority of self-referrals to emergency departments (ED) are not appropriate patients for emergency units. ${ }^{29,38-40}$

Ideally, Nigerian health system is a tripartite structure comprising of primary, secondary and tertiary healthcare facilities with intercalated referral pathway. ${ }^{41}$ Though emergency cases has no restricted access to the ED, the high numbers of self-referrals and referrals from $\mathrm{CHOP}$ highlights the non-coordinated interaction between the three levels of care. Hence a tertiary care facility like ours, which is supposed to concentrate on managing critical and complex cases, is being overburdened. Research had identified three factors that can lead to overcrowding of ED as: input factors, throughput factors and output factors. ${ }^{27-29}$ Primary and secondary health care facilities should be strengthened in order to reduce self-referral to tertiary centres in the Country. We urge the implementation of Universal Health Coverage to improve patients and families' access to early care

Our study revealed that comparable proportion (43.8\%) of children were admitted during the morning shift as compared to a report of $52 \%$ by Bashkin et al in Isra$\mathrm{el}^{27}$ Majority of mothers in Calabar metropolis are either civil servants or self-employed and may only notice the acute symptoms in their children after close of business. Hence three-fifth of emergency cases presented at offhour. Such patterns of presentation suggest the need for the availability of more senior medical staff during offhours in CHER.

Similar to previous studies from LMICs, the common diagnoses in this study (respiratory tract infections (53.7\%), malaria (52.4\%), septicaemia (14.3\%) and complications of SCA $(8.5 \%)$ ) were preventable illnesses. ${ }^{8,10-12}$ Therefore, public health campaign on prevention and treatment of common infectious diseases and premarital counselling against SCA should be intensified. Compared with reports of previous authors in the same centre, the trends in the prevalence of meningitis increased from $0.7 \%$ in 1988 (42) to $2.7 \%$ in $2012^{22}$ and then $10.3 \%$ in this present study. The current prevalence of meningitis at our centre is also higher than reports of $2.2 \%$ and $5 \%$ from Asaba $^{24}$ and Umuahia ${ }^{13}$ respectively in Southern Nigeria. The reason for this increased meningitis trend is not readily apparent but overcrowding with poor ventilation due to increased urban migration and the impact of global warming and desertification may be contributory. ${ }^{43} \mathrm{Al}$ though our region (Southern Nigeria) lies outside the traditional meningitis belt, this pattern may suggest an impending regional epidemic. A further public health research on prevalence of meningitis is warranted. We also suggest that mass administration of cerebrospinal meningitis vaccination should be considered in this region. 


\section{Predictors of hospital stay}

Bench marked by our unit's policy of maximum stay of 72 hours, about $16 \%$ of the children had prolonged hospital stay (PHS). Although Bekmezian et $\mathrm{al}^{28}$ also reported a similar rate of prolonged stay of $13 \%$, their cut off was shorter ( $>8$ hours). We hypothesize that earlier discharge of majority of participants were in part due to early review of most patients within 15 minutes of arrival at the CHER. Timely review by the unit Consultant and other appropriate Paediatric subspecialties might have also contributed to the relatively low rate of prolonged hospital stay in our centre. In addition, about two-thirds of the patients received intervention within 30 minutes of arrival at CHER.

We found from multivariable analysis, that the diagnosis (in descending order of SCA, malaria, sepsis/meningitis and respiratory illness), had higher odds of prolonged hospital stay as compared to surgical cases. This suggests that prevention and prompt management of infectious diseases can reduce overcrowding of CHER in our environment. Since patients' relations pay out of pocket for the care of their wards, parents of children presenting with the aforementioned diagnosis should be counselled early about the possibility of prolonged stay. Kuti at al proposed that such counselling can reduce frustration, engender confidence by guardians and prevent the risk of discharging against medical advice. ${ }^{44}$ Indeed, public health intervention to prevent sickle cell anaemia is of essence. Furthermore, parents of children with sickle cell anaemia should be counselled on how to prevent and manage complications of the disease. Routine early haemoglobin genotyping should be encouraged as most parents usually do not know the genotype of their children prior to being admitted for complications of sickle cell anaemia.

We found that children that had alternative care before presentation had the longest mean hospital stay which is more than doubled the mean hospital stay of those children that had no prior treatment $(216.0 \pm 0.0 \mathrm{Vs}$ $78.5 \pm 81.5)$. It is therefore imperative that mothers should be counselled on appropriate home treatment for common ailments. We expected that there may be worsening prognosis if mothers delay in bringing their children to the hospital. Surprisingly, children that had prior multiple treatments, or that visited another health facility before presentation had $59 \%$, and $61 \%$ lesser chance of staying beyond 72 hours as compared to children that did not. However, this pattern may suggest that such children had appropriate treatment at those primary or secondary facilities. Appropriate counselling would have prevented their further presentation at a tertiary centre as ours. Nonetheless, our finding may also suggest that the lower level medical centres referred the children promptly for acute care at our centre, (possibly because of lack of personnel and infrastructure at their health facility. ${ }^{4}$ Aggressive care of such referred cases may lead to early recovery of the children. Similarly, Bekmezian at al found that children that were promptly transferred to Intensive Care Units and those that had pulse oximeter in an ED were discharged early. ${ }^{28}$ Perhaps, the children had effective and appropriate home remedy or institutional care but the mothers still sought help at our centre because of anxiety. Studies have shown that children that arrived at emergency departments during off hours may stay longer. ${ }^{12,23,28,45}$ This is because highly skilled personnel and some investigations may not be available at such period of the day. However, our study revealed that there was no statistically significant difference in the length of stay of the children based on the period of admission. Thus, the usual slowdown of services during off hour appeared not to have affected hospital stay at our centre. Although, infants are usually more vulnerable to infectious diseases as compared to older children, ${ }^{11}$ we found that there was no statistically significant difference in the odds of prolonged stay and the age of our study participants. This may be related to the high vaccination rate (99.1\%) among our cohort of participants, which improved the prognosis of common infectious diseases among the infants.

It was expected that the cadre of admitting medical staff should affect the length of hospital stay of the children. Although our results from the Analysis of Variance and multivariable analysis suggest that there may be an increasing length of stay with increasing length of experience of admitting staff, this relationship was not statistically significant. This pattern might be because consultants preferentially see the very ill children (that may eventually stay longer,) based on the triage protocol of the unit.

Similar studies from other climes showed that varying predictors impact hospital stay, suggesting that the socioeconomic and health system milieu of each region may play significant roles. For example, Chaou et $\mathrm{al}^{30}$ and ChungHsien et $\mathrm{al}^{31}$ in Chang Gung, Taiwan found in contrast to our study that increased age, day-shift or weekend arrival, 
and gender had statistically significant impact on stay at paediatric EDs. The risk factors that we studied may be different from what was considered by previous studies from other regions because of differences in organisation of health systems. For example, Wiler et $\mathrm{al}^{32}$ in USA, studied the impact of ambulance diversion on hospital stay which was not considered by us while in a paediatric $\mathrm{ED}, \mathrm{Li}$ et $\mathrm{al}^{33}$ found that delayed hospitalization greater than 16 hours had the longest LOS.

\section{Hospital interventions and outcomes}

More than two-thirds $(67.9 \%)$ of the patients received intervention within 30 minutes of arrival. This is a desirable service delivery indicator as a timely delivery of emergency care services can build confidence in care givers and improve patients' outcome. ${ }^{46}$ The consensus document of the society for Academic Emergency Medicine recommended morbidity specific time to intervention ranging from $<10$ minutes to 6 hours. This study finding falls well within this irrespective of pathology. ${ }^{47}$

At discharge, majority of the children received antibiotics that were mostly commenced empirically, as only $22 \%$ of antibiotic administration was guided by blood culture results. This calls for development of protocol on judicious prescription of antibiotics to avoid community acquired antibiotic resistance. Our study revealed that, $4.8 \%(n=40)$ of the total admissions were transfused on account of severe malaria $(n=31 ; 77.5 \%)$ and SCA $(n=)$; $22 \%$ ). Our finding is similar to an earlier study from our environment ${ }^{42}$ in which severe malaria and SCA were the major indications for blood transfusion. However, the transfusion rate in this study was lower than the transfusion rate in 1988 (4.8\% Vs 11.2\%). ${ }^{42}$ The lower blood transfusion rate may be due to the wide spread use of Artemisinin combination therapies (ACT), coupled with the use of Long Lasting Insecticide treated bed net (LLITN) and better in-hospital and follow-up management of SCA. Recognising the multiple complications of blood transfusions, we suggest that efforts at further reducing the current transfusion rates should focus on counselling mothers on prevention of severe malaria and complications of SCA.

The proportion of properly disposed children in this study was slightly higher than what was obtained in 2012(82.8\% Vs $71.8 \%$ ). ${ }^{22}$ This might partly suggest that there was an overall improved care at the unit. Furthermore, there has been a progressive decline in mortality rate at our centre from $5 \%$ in $1988(42)$ to $2 \%$ in $2012^{22}$ and $0.5 \%$ in this present study, that was conducted in 2014. This decreasing trend may further suggest an improvement in the overall care offered at the centre. A dedicated paediatric consultant at the unit, coupled with a review of the triage system (the first on call has been changed from a house officer to a junior resident), and the weekly clinical audit meeting could have contributed to the downward mortality trend. In contrast; many CHER in Nigeria had higher mortality rates $(2-17.5 \%)$. During similar study period. ${ }^{2,11,12,15,18,21-23}$

A review of the clinical course of the children that died showed that they had preventable conditions. They presented later than two weeks after onset of illness and died within 48 hours of admission before resuscitative measures could impact on their prognosis. Unfortunately, autopsy which could have improved our practice and knowledge of the conditions was not done despite counselling because of poor perception and cultural believes of the parents.

\section{Limitation of the study}

This is an institutional based cross sectional study and we may not be able to be certain about causality in the associations demonstrated. More so, there may be other residual confounding variables that might not have been considered. Nevertheless, we still believe that this pioneer study has contributed invaluable evidence to improving the operations of paediatric emergency care in Nigeria and sub Saharan Africa. The principal investigator randomly cross checked the data extraction of the resident doctors by comparing the records in the proforma with the clinical case files of the participants and did not observe any significant error. However, since the information extracted are fixed figures that do not require the opinion or judgement of the extractor, we did not calculate the inter-rater score. ${ }^{48}$

\section{Conclusion}

Only about $16.4 \%$ of our participants had prolonged hospital stay beyond 72hours. Children with common infectious diseases (such as Malaria and sepsis/meningitis) and haemoglobinopathies (SCA) had higher risk of prolonged hospital stay beyond 72 hours. Furthermore, children who had alternative herbal remedy before presentation had the highest hospital stay Thus, Public enlightenment campaign to prevent complications of com- 
mon infectious conditions such as malaria, sepsis and meningitis and haemoglobinopathies is very important. Inappropriate pre-hospital treatments should also be discouraged. Our study highlighted that majority of patients were self-referrals and presented at off hours. This calls for health system strengthening of the primary and secondary healthcare facility of Nigeria to reduce overcrowding of the tertiary care centre like the site of the present study. We urge the implementation of Universal Health Coverage to improve patients and families' access to early care and adoption of high level manpower as first line doctor in CHER as this will assure quality services and positively impact patient's outcomes. In addition, government effort should be geared towards training more Paediatric Emergency specialist, and provisions of essential hospital infrastructures to further reduce morbidity and mortality.

\section{Acknowledgement}

The authors acknowledge all the participants and their care givers for participating in this study. Also credit goes to all the Residents doctors and Interns in UCTH that assisted in data collection.

\section{Declaration of conflict of interest}

The authors declare no conflict of interest that requires disclosure.

\section{Funding sources/disclosures}

The corresponding author is not a recipient of any form of scholarship for this paper.

\section{References}

1. Molyneux E RA. Emergency Medicine in differently resourced settings: what can we offer each other? Emerg Med $J$ [Internet]. 2002;19(5):378-9. Available from: https:// www.ncbi.nlm.nih.gov/pmc/articles/PMC1725935/ pdf/v019p00378.pdf

2. Bassey EU, Ijezie E. Pediatric Emergencies Seen in a Tertiary Hospital in Uyo , Akwa Ibom State of Nigeria: A two Year Review. 2016;4(4):42-5.

3. ElizabethMolyneux. Paediatric emergency care in developing countries. Lancet. 2000;356:250-1.

4. Obermeyer Z, Abujaber S, Makar M, Stoll S, Kayden SR, Wallis LA, et al. Emergency care in 59 low- and middle-income countries: a systematic review. Bull World Health Organ. [Internet]. 2015;93(October 2014):577586G. Available from: http://www.who.int/bulletin/volumes/93/8/14-148338/en/
5. Kissoon N, Goldman RD. Pediatric emergency medicine: A world of potential. Can J Emerg Med. 2007;9(6):453-5.

6. NHS England (London Region). Children and young people's health services in London/ A case for change. Strategic Clinical Networks. NHS England (London Region) [Internet]. 2013. Available from: http://www.londonscn.nhs.uk/wp-content/uploads/2014/12/londoncyp-scn-caseforchg-122014.pdf

7. Riccardo Longhi, Raffaella Picchi, Domenico Minasi ADCM. Pediatric emergency room activities in Italy: a national survey. Ital J Pediatr. 2015;41:77.

8. Naghavi M, Wang H, Lozano R, Davis A, Liang X, Zhou M, et al. Global, regional, and national age-sex specific all-cause and cause-specific mortality for 240 causes of death, 1990-2013: A systematic analysis for the Global Burden of Disease Study 2013. Lancet [Internet]. Elsevier Ltd; 2015;385(9963):117-71. Available from: http://dx. doi.org/10.1016/S0140-6736(14)61682-2

9. Choonara I.Children's medicines - a global problem. Arch Dis Child [Internet]. 2009;94(6):467. Available from: http://adc.bmj.com/content/archdischild/94/6/467. full.pdf

10. Ibeziako S, Ibekwe R. Pattern and Outcome of Admissions in the Children's Emergency Room of the University of Nigeria Teaching Hospital, Enugu. Niger J Paediatr [Internet]. 2002;29(4):103-8. Available from: http:// www.ajol.info/index.php/njp/article/view/12018

11. Aminu M, Bassey E, Bilkisu G, Muyideen A, Smart A, Sunday O. Pattern of medical childhood morbidity and mortality in a new specialist hospital in Gusau, Nigeria. Ann Niger Med. [Internet]. 2014;8(1):15. Available from: http://www.anmjournal.com/text. asp?2014/8/1/15/141024

12. Anyanwu O, Ezeanosike O, Ezeonu C. Pattern and outcome of admissions at the children emergency room at the Federal Teaching Hospital Abakaliki. African J Med Heal Sci [nternet]. 2014;13(1):6. Available from: http:// www.ajmhs.org/text.asp?2014/13/1/6/139435

13. Charles NC, Chuku A, Anazodo NM. Childhood mortality in federal medical centre Umuahia, South Eastern Nigeria. Oman Med J. 2014;29(5):320-4.

14. Iloh GUP, Ofoedu JN, Njoku PU, Amadi AN, Godswill-Uko EU. The magnitude of under-five emergencies in a resource-poor environment of a rural hospital in eastern Nigeria: Implication for strengthening the household and community-integrated management of childhood illnesses. N Am J Med Sci. 2012;4(8):344-9. 
15. Ndukwu CI, Onah SK. Pattern and outcome of postneonatal pediatric emergencies in Nnamdi Azikiwe University Teaching Hospital, Nnewi, South East Nigeria. Niger J Clin Pract. 2015;18(3):348-53.

16. Mohammed S, Islam S. Globalization and Health Non-communicable Diseases ( NCDs ) in developing countries : a symposium report. 2014;

17. Calvello EJB, Tenner AG, Broccoli MC, Skog AP, Muck AE, Tupesis JP, et al. Operationalising emergency care delivery in sub-Saharan Africa: consensus-based recommendations for healthcare facilities. Emerg Med J. [Internet]. 2016;33(8):573-80. Available from: http://emj. bmj.com/lookup/doi/10.1136/emermed-2015-204994 18. Forae GD, Uchendu OJ, Igbe AP. An audit of paediatric mortality patterns in a Nigerian teaching hospital. Niger MedJ. [Internet]. 2014;55(2):130-3. Available from: http:/ / www.ncbi.nlm.nih.gov/pubmed/24791046\%5Cnhttp:// www.pubmedcentral.nih.gov/articlerender.fcgi?artid=PMC4003715

19. Boutayeb A, Boutayeb S. The burden of non communicable diseases in developing countries. Int J Equity Health [Internet]. 2005;4(1):2. Available from: http://equityhealthj.biomedcentral.com/articles/10.1186/1475-9276-4-2

20. Hu FB. Globalization of diabetes: The role of diet, lifestyle, and genes. Diabetes Care. 2011;34(6):1249-57.

21. Okoronkwo NC. Pattern ofmorbidity and mortality of childhood illnesses at the children emergency room of Abia State University Teaching Hospital, Aba, Nigeria. East Cent Africa Med J. 2015;2:70-3. PubMed

22. Enyuma C, Ikpeme O, Brown-Abang E, Uket H. Morbity and mortality in a Children Emergency Room in Calabar. J Med Africa. 2016;1(1):6-10. PubMed

23. Adeboye M a, Ojuawo a, Ernest SK, Fadeyi a, Salisu OT. Mortality pattern within twenty-four hours of emergency paediatric admission in a resource-poor nation health facility. West Afr J Med. [Internet]. 2010;29(4):249_ 52. Available from: http://www.ncbi.nlm.nih.gov/ pubmed/20931513

24. Ezeonwu B, Chima O, Oguonu T, Ikefuna A, Nwafor I. Morbidity and mortality pattern of childhood illnesses seen at the children emergency unit of federal medical center, Asaba, Nigeria. Ann Med Health Sci Res. [Internet]. 2014;4(9):239. Available from: http://www.amhsr.org/ text.asp?2014/4/9/239/141966

25. Okechukwu AA. Morbidity pattern in paediatric outpatient unit of the University of Abuja Teaching Hospital, Gwagwalada, Nigeria. 2010;13(March):1-6.
26. NI Paul, Edelu B. Evaluation of the preparedness of the children's emergency rooms (CHER) in Southern Nigeria for service delivery. Niger J Paediatr. [Internet]. 2017;44(2):63-7. Available from: https://www.ajol.info/ index.php/njp/article/viewFile/158192/147793

27. Bashkin O, Caspi S, Haligoa R, Mizrahi S, Stalnikowicz R. Organizational factors affecting length of stay in the emergency department: initial observational study. Isr J Health Policy Res [Internet]. Israel Journal of Health Policy Research; 2015;4(1):38. Available from: http://www.ijhpr. org/content/4/1/38

28. Bekmezian A, Chung PJ, Cabana MD, Maselli JH, Hilton JF, Hersh AL. Factors associated with prolonged emergency department length of stay for admitted children. Pediatr Emerg Care. 2011;27(2):110-5.

29. Young-Harry ON, Dienye PO, Diete-Spiff KO. Pattern of inappropriate cases presenting to the accident and emergency department in a Nigeria tertiary hospital. South African Fam Pract [Internet]. Cogent, 2015;57(4):2538. Available from: http://dx.doi.org/10.1080/20786190. 2014.978114

30. Chaou C-H, Chen H-H, Chang S-H, Tang P, Pan S-L, Yen AM-F, et al. Predicting Length of Stay among Patients Discharged from the Emergency Department-Using an Accelerated Failure Time Model. Fernandez-Reyes D, editor. PLoS One [Internet]. Public Library of Science; 2017 Jan 20 [cited 2018 Aug 17];12(1):e0165756. Available from: http://dx.plos.org/10.1371/journal.pone.0165756 31. Chaou C-H, Chiu T-F, Yen AM-F, Ng C-J, Chen H-H. Analyzing Factors Affecting Emergency Department Length of Stay-Using a Competing Risk-accelerated Failure Time Model. Medicine (Baltimore) [Internet]. Wolters Kluwer Health; 2016 Apr [cited 2018 Aug 17];95(14):e3263. Available from: http://www.ncbi.nlm. nih.gov/pubmed/27057879

32. Wiler JL, Handel DA, Ginde AA, Aronsky D, Genes NG, Hackman JL, et al. Predictors of patient length of stay in 9 emergency departments. Am J Emerg Med. [Internet]. 2012 Nov [cited 2018 Aug 17];30(9):1860-4. Available from: http://www.ncbi.nlm.nih.gov/pubmed/22633732 33. Li S-T, Chiu N-C, Kung W-C, Chen J-C. Factors Affecting Length of Stay in the Pediatric Emergency Department. Pediatr Neonatol [Internet]. 2013 [cited 2018 Aug 17];54:179-87. Available from: http://dx. doi.org/10.1016/j.pedneo.2012.11.014Availableonlineatwww.sciencedirect.comjournalhomepage:http:/ /www. pediatr-neonatol.com

34. Tuffin H, Twomey M WL. Policy for implementation 
of the Triage of Patients in Western Cape Emergency Centers. In: T W, editor. Emergency Medicine Guidelines for the Western Cape. 2013th ed. cape Town: Eclipse print, 2013. p. 4-13.

35. Okechukwu AA, Nwalozie C. Morbidity and mortality pattern of admissions into the Emergency Paediatric Unit of University of Abuja Teaching Hospital, Gwagwalada. Niger J Med. 2011;

36. Tumwesigire S, Watson S. Health seeking behavior by families of children suspected to have malaria in Kabale: Uganda. Afr Health Sci. 2002;2(3):94-8.

37. Mbagaya GM, Odhiambo MO, Oniang'o RK. Mother's health seeking behaviour during child illness in a rural Western Kenya community. Afr Health Sci. 2005;5(4):3227.

38. van der Linden MC, Lindeboom $\mathrm{R}$, van der Linden $\mathrm{N}$, van den Brand CL, Lam RC, Lucas C, et al. Self-referring patients at the emergency department: appropriateness of ED use and motives for self-referral. Int J Emerg Med. [nternet]. 2014;7(1):28. Available from: http://intjem. springeropen.com/articles/10.1186/s12245-014-0028-1 39. Kraaijvanger N, Rijpsma D, Van Leeuwen H EM. Self-referrals in the emergency department: reasons why patients attend the emergency department without consulting a general practitioner first-a questionnaire study. Int J Emerg Med [Internet]. International Journal of Emergency Medicine; 2015;8(1):1-6. Available from: http://0-www.springer.com.lib.exeter.ac.uk/medicine/critical + care +and +emergency + medicine/journal/12245\%5Cnhttp://0-ovidsp.ovid.com.lib.exeter. ac.uk/ovidweb.cgi? $\mathrm{T}=\mathrm{JS} \& \mathrm{PAGE}=$ reference $\& \mathrm{D}=$ emed1 $7 \& N E W S=N \& A N=607162092$

40. Kraaijvanger N, van Leeuwen H, Rijpsma D, Edwards M. Motives for self-referral to the emergency department: a systematic review of the literature. BMC Health Serv Res [Internet]. BMC Health Services Research; 2016;16(1):685. Available from: http://dx.doi.org/10.1186/s12913-016$1935-\mathrm{z}$
41. World Health Organization. The Nigerian Health System [Internet]. Health Systems. 2014. p. 21-30. Available from: http://www.who.int/pmnch/countries/nigeria-plan-chapter-3.pdf

42. Antia-Obong OE. Paediatric Emergencies in Calabar. Nig Med Pr. 1992;23(51-5).

43. Molesworth AM, Cuevas LE, Connor SJ, Morse AP, Thomson MC. Environmental risk and meningitis epidemics in Africa. Emerg Infect Dis. 2003;9(10):1287-93. 44. Kuti BP, Adegoke SA, Oyelami OA, Ota MO. Predictors of prolonged hospitalisation in childhood pneumonia in a rural health centre. South African J Child Heal. [nternet]. 2014 Feb 5 [cited 2018 Aug 17];8(1):11. Available from: http://hmpg.co.za/index.php/sajch/article/ view $/ 736$

45. Wammanda RD, Ali FU. Conditions associated with the risk of death within 24 hours of admission in children in Zaria, Nigeria. Ann Afr Med. 2004;3(3):134-7.

46. Welch SJ, Asplin BR, Stone-Griffith S, Davidson SJ, Augustine J, Schuur J, et al. Emergency Department Operational Metrics, Measures and Definitions: Results of the Second Performance Measures and Benchmarking Summit. Ann Emerg Med. [Internet]. 2011 Jul [cited 2018 Aug 17];58(1):33-40. Available from: http://www.ncbi. nlm.nih.gov/pubmed/21067846

47. Handel D, Epstein S, Khare R, Abernethy D, Klauer $\mathrm{K}$, Pilgrim R, et al. Interventions to Improve the Timeliness of Emergency Care. Acad Emerg Med. [Internet]. 2011 Dec [cited 2018 Aug 17];18(12):1295-302. Available from: http://www.ncbi.nlm.nih.gov/pubmed/22168194 48. Gilbert EH, Lowenstein SR, Koziol-McLain J, Barta DC, Steiner J. Chart Reviews In Emergency Medicine Research: Where Are The Methods? Ann Emerg Med. [Internet]. Mosby; 1996 Mar 1 [cited 2018 Aug 17];27(3):305-8. Available from: https://www.sciencedirect.com/science/ article/pii/S0196064496702640 